\title{
Wide-field four-channel fluorescence imager for biological applications
}

\author{
Madhuri Thakur \\ Rice University \\ Department of Chemical and Biomolecular Engineering \\ 6100 South Main Street \\ Houston, Texas 77005
}

Dmitry Melnik

The Ohio State University

Department of Chemistry

100 West 18th Avenue

Columbus, Ohio 43210

\author{
Heather Barnett \\ The University of Washington \\ Department of Medicine \\ Seattle, Washington 98195 \\ Kevin Daly \\ Princeton University \\ Department of Chemical Engineering \\ Princeton, New Jersey 08544
}

Christine H. Moran

Washington University

Department of Biomedical Engineering

One Brookings Drive

St. Louis, Missouri 63130

\section{Wei-Shun Chang \\ Stephan Link}

Christopher Theodore Bucher

Carter Kittrell

Robert Curl

Rice University

Department of Chemistry

6100 South Main Street

Houston, Texas 77005

\begin{abstract}
A wide-field four-channel fluorescence imager has been developed. The instrument uses four expanded laser beams to image a large section $(6 \mathrm{~mm} \times 9 \mathrm{~mm})$. An object can be sequentially illuminated with any combination of 408-, 532-, 658-, and 784-nm lasers for arbitrary (down to $1 \mathrm{~ms}$ ) exposure times for each laser. Just two notch filters block scattered light from all four lasers. The design approach described here offers great flexibility in treatment of objects, very good sensitivity, and a wide field of view at low cost. There appears to be no commercial instrument capable of simultaneous fluorescence imaging of a wide field of view with four-laser excitation. Some possible applications are following events such as flow and mixing in microchannel systems, the transmission of biological signals across a culture, and following simulations of biological membrane diffusion. It can also be used in DNA sequencing by synthesis to follow the progress of the photolytic removal of dye and terminator. Without utilizing its time resolution, it can be used to obtain four independent images of a single tissue section stained with four targeting agents, with each coupled to a different dye matching one of the lasers. () 2010 Society of Photo-Optical Instrumentation Engineers. [DOI: 10.1117/1.3374052]
\end{abstract}

Keywords: imaging systems; fluorescence microscopy.

Paper 09253R received Jun. 17, 2009; revised manuscript received Jan. 18, 2010; accepted for publication Feb. 11, 2010; published online Apr. 2, 2010.

\section{Introduction}

The field of fluorescence imaging for biomedical applications has undergone a dramatic expansion over the past 20 years. It is the most rapidly expanding technique employed today, in both the medical and biological sciences, ${ }^{1-9}$ a fact that is spurring the development of more sophisticated microscopes ${ }^{10-14}$ and wide-field imagers. ${ }^{15}$ The availability of hundreds of fluorescent labels ${ }^{16,17}$ with known excitation and emission curves and well-understood biological structure targets has accelerated the application of fluorescence microscopy in both clinical laboratories and research. These developments allow great

Address all correspondence to: Robert Curl, Rice University, Chemistry Department, 6100 South Main Street, Houston, Texas 77005. Tel: 713-348-4816; Fax: 713-348-5155; Email: rfcurl@rice.edu flexibility and specificity in what can be imaged. Many fluorescent dyes are compatible with living organisms, making in vivo imaging possible. In particular, functional imaging of a biological process can be achieved ${ }^{3-5,18}$ by selecting a fluorescent label that attaches to a dynamic structure of interest within the sample.

Imager development has more than kept pace with the development of fluorophores. Instruments with exquisite resolution are readily available commercially. For wide-area applications, scanners, which work very well and are capable of scanning over an area of several square centimeters with extremely high resolution, are commercially available. Recently, Sevick-Muraca has developed an extremely wide field, lowresolution instrument using deep-red $(780 \mathrm{~nm})$ excitation and

1083-3668/2010/15(2)/026016/9/\$25.00 @ 2010 SPIE 


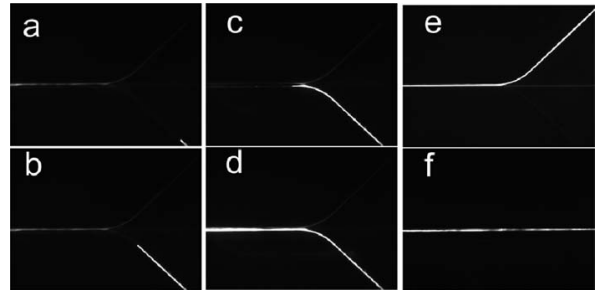

Fig. 1 Fluorescence images of labeled tetraethylene glycol flowing through a microchannel system. Panels (a) to (d) $10^{-3} \mathrm{M}$ coumarin 515 excited by the 408-nm laser. (a) Initial dye fluorescence appearance (exposure time $10 \mathrm{~ms}$ ); (b) exposure $4 \mathrm{~s}$ later (exposure time $10 \mathrm{~ms}$ ); (c) exposure $4 \mathrm{~s}$ later (exposure time $10 \mathrm{~ms}$ ); (d) exposure $4 \mathrm{~s}$ later (exposure time $10 \mathrm{~ms}$ ); (e) fluorescence from $10^{-3} \mathrm{M}$ Rh6G labeled dye excited by 532-nm laser flowing through another channel; and (f) fluorescence from $10^{-3} \mathrm{M}$ LD700 dye excited by 658-nm laser.

used it to make movies of flow in the lymph system. ${ }^{15}$

Major microscope manufacturers offer fluorescence microscopes that provide a wide choice of objectives and eyepieces, permitting the user to observe with a wide field of view and low resolution or a narrow field of view and high resolution. In these microscopes, fluorescence excitation is provided in epi-illumination by means of a dichroic mirror placed in the region between the objective and the tube lens using lamp illumination with the excitation wavelength region selected by appropriate filters.

For applications where a wide field, high resolution, and high sensitivity are required, the well-known axiom of microscopy that wide field of view and high spatial resolution cannot be achieved in the same instrument is overcome by fluorescence scanners that sequentially observe fluorescence from a series of tiny spots and rebuild the image from these observations.

Even with this variety of commercial instruments, applications do exist that cannot be served by choosing a suitable instrument. In particular, if simultaneous observation of multilaser excited fluorescence from a relatively large field of view with high sensitivity is required, the instrument simply does not exist. Conventional fluorescence microscopes set for low magnification sacrifice sensitivity for field of view in order to avoid vignetting. In the infinity focus region between objective and tube lens, the collimated light from off-axis points does not travel down the tube axis, and if a point is far enough from the optic axis, part of the light from it is lost. Thus, to obtain a wide field of view without significant vignetting the working distance is made long with a consequent loss of numerical aperture. Turning aside from conventional microscopes, scanning imagers cannot observe fluorescence from well-separated points simultaneously nor are they intended to; however, there are situations where such observations are desirable.

Figure 1 illustrates the capability this microscope has that scanning imagers lack. Figures 1(a)-1(d) show the entry of dye excited by the violet laser entering and passing through a microfluidics channel with the time lapse between images $\sim 4$ s. Figure 1(e) shows another channel on the same chip filled with dye excited by a green laser, and Fig. 1(f) shows yet another channel filled with dye excited by a red laser. A scanning imager can cover a larger area than this with much better resolution, but it would be incapable of capturing the fluid flow.

The imager reported here was designed to support a project for DNA sequencing by synthesis where the imager would have the function of following in time the removal of dye from a microarray by UV photolysis while water flushing. After this process is completed and new dye-labeled bases have been appended, then the imager would read fluorescence from the microarray without the necessity of moving it.

The instrument could prove useful in surveying tissue sections for fluorescent markers with sensitivity limited only by light scattering from the tissue. Another possible example would be investigation of enzyme reaction kinetics in a multiplexed manner by following the production of fluorescent products or by the attachment of two dyes, thus creating fluorescence resonance energy transfer (FRET). Another is the study of diffusion of dye in a lipid bilayer. This instrument provides more flexibility in deployment than scanning microscopes and a simultaneous view of the system. It provides higher sensitivity than conventional wide-field microscopes and has multiple-laser excitation capability.

\section{Imaging System Overall Design}

Vignetting is rarely an issue in microscope design but is ever present in the design of camera lenses. Thus, the choice was made to use camera lenses in the construction of this imager. In developing a fluorescence imager design, in addition to avoiding vignetting, another issue arises in the treatment of rays emanating from the extreme off-axis points of the object. Laser light scattered from the object must be blocked using notch filters, and the performance of these filters is degraded for off-normal rays.

A simple design approach (a) for the desired imager is to set two infinity-focused camera lenses facing each other with the notch filters to block scattered laser light between them. Behind one lens is the object, and behind the other is a charge-coupled device (CCD) to capture the image. Although the collimated light from an extreme off-axis point of the object strikes the notch filters off-normal, opening the possibility of degraded filter performance, and strikes the second camera lens off-axis, guaranteeing that there will be some vignetting, in practice this simple design provides decent performance. And it does so at quite low cost through the use of an inexpensive cooled CCD camera for amateur astronomers and the use of relatively inexpensive lasers. However, any further separation of the two lenses, such as would be required for epi-illumination using a dichroic mirror, is not feasible because it will introduce too much space in the infinity focus region with resulting serious vignetting.

In addition to the simple design in which two infinityfocused camera lenses face each other, we have explored a different approach (b) in which an intermediate image plane is introduced where two half-field lenses sandwich the notch filters. The field lens redirects the rays from this intermediate image into the second lens, greatly reducing vignetting. By splitting the field lens and placing the notch filters between the two halves of the field lens, the angle at which the rays strike the filters is brought closer to the perpendicular, thereby reducing degradation of notch filter performance. 


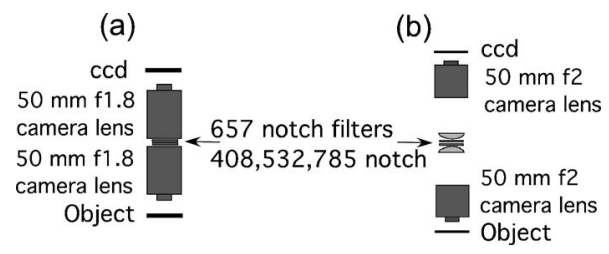

Fig. 2 Imager configurations: (a) simpler configuration; (b) intermediate image configuration.

Figure 2 shows schematics of both wide-field four-channel fluorescence imager designs. Common to both designs are fluorescence excitation lasers arranged to illuminate the object plane, a camera lens serving as the collecting objective, an intermediate region where notch filters to block scattered laser light are placed, and a second camera imaging lens focusing the fluorescence onto a cooled CCD camera. The lasers and camera are controlled by software developed for this purpose. This highly flexible software also carries out both preprocessing of the images and in some cases their ultimate analysis.

The field of view of this wide-field-of-view imager is limited in one-to-one imaging by the dimensions $(8.98$ by $6.71 \mathrm{~mm}$ ) of our inexpensive cooled CCD (1.4 million 6.45 $\times 6.45 \mu \mathrm{m}$ pixels). As you will see, resolution is limited by pixel size; CCDs with smaller pixels (currently down to about $2 \mu \mathrm{m})$ exist, offering an avenue to modestly higher resolution. The simple camera lenses facing each other in this design [Fig. 2(a)] could be extended to larger fields of view if a larger CCD and larger lenses were introduced.

The components of the camera are now described in detail.

\subsection{Optical Train}

The camera lenses chosen were 50-mm focal length Fujinon (HF50SA-1) f1.8 CCTV lenses. Considerably faster camera lenses are readily available, but these tend to have shorter focal lengths or are much more expensive. Short focal length lenses are not suitable because the rays from extreme off-axis points travel through the system at steeper angles, leading to both vignetting and degradation of filter performance. One notch filter (Semrock NF01-785U \$2600) blocks scattered light from three lasers: $408 \mathrm{~nm}, 532 \mathrm{~nm}$, and $784 \mathrm{~nm}$. A second notch filter (Mkphotonics \$1500) blocks scattered light from the 658-nm laser. The optical train in Fig. 2(a) is so simple that further description is not required. The more elaborate optical train in Fig. 2(b) with an intermediate image does merit further description. The field lenses used were a pair of 200-mm focal length, 50-mm-diam achromats (Edmund Optics NT45-1792). The amount of magnification is limited by the size of available filters; the largest readily available commercial filters are $50 \mathrm{~mm}$ in diameter. Since the CCD has a diagonal dimension of $11.2 \mathrm{~mm}$, this implies a maximum magnification of 4.46. It is not possible to achieve sharp focus with a magnification (viewed from the point of view of the intermediate image) as small as 4.46 with this lens set to its shortest focal length when the object is placed at the normal working distance from the rear of the lens. By adding a 2-diopter close-up lens to each lens and increasing the rear working distances by $5 \mathrm{~mm}$, sharp focus was achieved at the cost of increasing the overall f\# to approximately 2 .

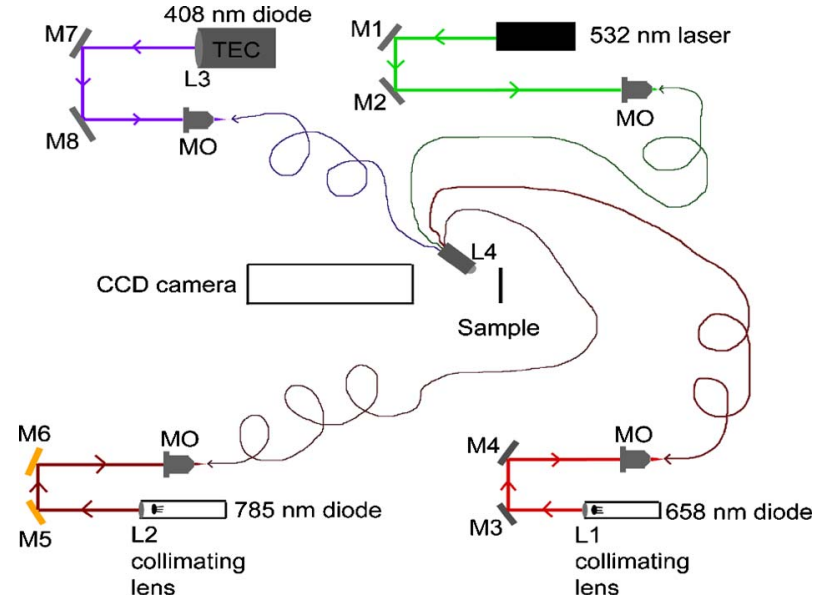

Fig. 3 Four-color optical fiber object illumination scheme.

\subsection{Flourescence Excitation Lasers}

The four exciting lasers have wavelengths of 408, 532, 658, and $784 \mathrm{~nm}$ with power outputs of $60,150,90$, and $120 \mathrm{~mW}$, respectively. The 408-nm laser is a $60-\mathrm{mW}$ Sanyo GaN diode (LS5017); the 532-nm laser is a Laserglow diode-pumped solid-state laser (doubled Nd: $\mathrm{YVO}_{4}$, Model LRS-532-TM50). (This laser was rated at $50 \mathrm{~mW}$, but produced nearly $150 \mathrm{~mW}$.) The 658-nm laser is a 90-mW Hitachi laser diode (HL6535MG), and the 784-nm laser is a 120-mW Sharp laser diode (GH0781JA2C). The most expensive laser is the 408-nm GaN, which cost over $\$ 2000$, but prices for this relatively new technology should be decreasing. The 532-nm $\mathrm{Nd}: \mathrm{YVO}_{4}$ laser cost just under $\$ 1000$. The cost of each of the two red lasers was much lower.

\subsection{Oblique Illumination}

The scheme chosen for illuminating the object with the lasers was an oblique one. Two approaches to illumination were tested. For the images shown here, the four laser beams were directed through space from the four corners of the object, illuminating it obliquely. However, we have set up and tested a fiber-optic-based illumination scheme. The beam quality of the 532-nm doubled $\mathrm{Nd}$ vanadate laser is excellent, making coupling it into a single-mode fiber relatively easy. The other three lasers are diode lasers, which behave as small extended sources, but it proved possible to collimate satisfactorily each of these diode lasers using aspheric collimating lenses and then to couple them into single-mode fibers. The output powers emerging from the fibers are $30 \mathrm{~mW}(408 \mathrm{~nm}), 45 \mathrm{~mW}$ $(532 \mathrm{~nm}), 50 \mathrm{~mW}(658 \mathrm{~nm})$, and $50 \mathrm{~mW}(784 \mathrm{~nm})$. The output ends of the four fibers are brought together, providing a convenient illumination scheme (Fig. 3). This fiber illumination adds to the cost, as the cost of the four precision couplers into the fibers amounted to over $\$ 4000$. Diode lasers with fiber pigtails are available, but their output powers available at design time were thought too low.

Total internal reflection fluorescence (TIRF) illumination should also be feasible and is probably the only option for illuminating weakly fluorescent material bound to a surface 
under a many-micron thickness of water, as the Stokes Raman emission from the $\mathrm{O}-\mathrm{H}$ (oxygen-hydrogen) stretch vibrations of water is very strong.

\subsection{Camera}

The image was captured using a Starlight Xpress SXV-H9 camera costing about $\$ 3000$. The camera uses a Sony ICX285AL progressive scan CCD, with $1392 \times 1040$ $\times 6.45 \mu \mathrm{M}$ pixels in a $8.98 \times 6.71 \mathrm{~mm}$ active area. The CCD has its maximum quantum efficiency (QE) of $66 \%$ at about $510 \mathrm{~nm}$. At $450 \mathrm{~nm}$, which is about the maximum of a typical violet dye, it is about $55 \%$. At $810 \mathrm{~nm}$, which is about the maximum of the reddest dye, its quantum efficiency is about $23 \%$. The CCD is themoelectrically cooled to $30^{\circ} \mathrm{C}$ below ambient.

The camera specifications indicate that readout error is $7 \mathrm{e}^{-}$typical, $12 \mathrm{e}^{-}$maximum. The well depth is quoted as $27,000 \mathrm{e}^{-}$. The output of the CCD is in arbitrary units (AU), with the maximum possible output $65,536\left(2^{16}\right)$. As negative signals are not allowed, zero signal is offset to about 1000 $\left(2^{10}\right)$ AU. We determined experimentally by a somewhat nonstandard, but sound, statistical method that $1 \mathrm{e}^{-}=3.2 \mathrm{AU}$. The dark frame saturation time is specified as greater than $100 \mathrm{~h}$. Tests of the camera found that it met these specifications. In fact, the pixel-to-pixel variation of dark count could be quantitatively assigned to readout noise with only about one pixel in a thousand giving a strikingly higher signal.

One drawback of the camera is that if there is intense fluorescence striking a single pixel of the $\mathrm{CCD}$, the electrons that pile up on that pixel spill over into adjacent pixels. Another is that its $\mathrm{A} / \mathrm{D}$ converter limits the readout time to about $3.7 \mathrm{~s}$ for full-frame download and $0.5 \mathrm{~s}$ for binned $4 \times 4$ downloads.

\subsection{Control and Image Processing Software}

The camera came with its own data acquisition software using a USB connection. However, this software could not handle the requirements for computer control of the laser on/off sequences and the corresponding camera exposure and reading sequences. Except for the camera driver itself, the software to control the lasers, manage the camera, and manipulate the images was written from scratch in the Python programming language. This open-source interpreter language has a wide base of community support, and many modules with useful functionalities are available. Having complete control of the software allows easy connection to image processing modules customized for a particular application.

The need for this can be understood when the issues arising from nonuniform illumination are considered. It is difficult to achieve uniform illumination over a wide field of view without being forced to clip the illumination beam(s) with a resulting loss of peak intensity. Therefore, the background must be acquired and divided into the signal image in order to obtain an unbiased image of the fluorescing features. The means by which this can be accomplished depends strongly on the nature of the sample. For the quantitative example used to characterize the instrument described in the following, the approach to background extraction is described in some detail.

\section{Signal and Sensitivity Expectations and Testing}

Commercial wide field of view fluorescence microscopes are, as mentioned earlier, widely available, but they have low numerical apertures. One of the principal aims here is to construct a much more sensitive wide field of view instrument. The numerical aperture of the imager is about 0.25 ( 0.28 configuration a; 0.25 configuration b) making the ratio of the solid angle to $4 \pi$ about 0.015 . Since most of the optics are antireflection (AR) coated, the expected transmission of the optical chain including filters off-notch is expected to be about 0.4. Using 532-nm excitation as an example, the quantum efficiency of the CCD is almost $60 \%$ near $600 \mathrm{~nm}$. Multiplying these factors, the expected fraction of the emitted fluorescence photons that will give rise to one $\mathrm{CCD} \mathrm{e}^{-}(3.2$ $\mathrm{AU})$ is approximately 0.006 , assuming that none of the notches of the filters significantly blocks fluorescence.

Background noise levels are determined by noise arising from scattered light and fluorescence from the substrate supporting the sample reaching the detector and by noise intrinsic to the detector. As noted earlier, the readout noise of the CCD is about $10 \mathrm{e}^{-}(\sim 30 \mathrm{AU})$. In addition, there can be noise associated with thermal electrons. For our cooled CCD camera, such thermal noise is not significant for 2-min exposures except for a few (86 out of over 1.4 million) "hot" pixels for which the count rises over 2000 AU. Experimentally, it turns out that the readout noise and thermal noise are often negligible in comparison with "laser on" light. Background with a laser on, as would be expected, depends strongly on the substrate and its cleaning.

As a check of whether that sensitivity was meeting expectations, fluorescence from 100-nm orange microspheres (Invitrogen F8800) obtained using this imager were compared with those from a sample scanning confocal fluorescence microscope. The result of this comparison was that the imager sensitivity (in configuration b) is about a factor of two smaller than anticipated. This is good agreement for a comparison of this type. The imager has sufficient sensitivity to detect a single 0.1-micron dye-labeled microsphere, provided that the background from the substrate is made small enough.

\section{Resolution}

The CCD pixel size is $6.4 \times 6.4 \mu \mathrm{m}$, and our expectation was that resolution would be limited by pixel size. Resolution was tested using a United States Air Force resolution test chart (USAF) slide (Fig. 4). Figure 4(a) is a normal image showing much of the pattern on the slide taken in configuration a, but without the insertion of the two filters. The corners of this image are darker, as a result of vignetting. Figure 4(b) shows a small part of the image in which the center of the USAF pattern has been expanded. The grainy appearance of the image results from its pixel structure. The numbers labeling the groups and their elements were no longer discernible for the groups smaller than 5 (groups 6 and 7), and even the numbers labeling the elements of group 5 are almost indiscernible. The numbers labeling elements 4.3 [clear in original; see Fig. 4(a)] and 5.6 and the groups 6 and 7 have been added to the image in post-processiong for purposes of orientation.

A key characteristic of resolution limited by pixel size is that the apparent quality of an image is determined by how 


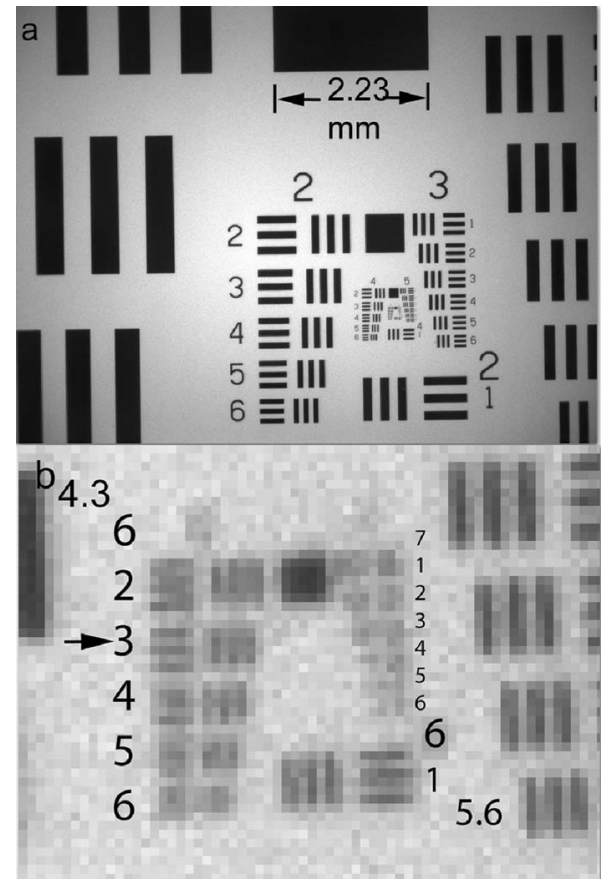

Fig. 4 (a) Central region of a USAF slide. This covers nearly the full size of the CCD (optical configuration a). (b) Expansion of part of Fig. 4(a). The horizontal bars of element 6.3 indicated by arrow has a width of 6.2 microns, which is almost the same width ( 6.4 microns) as the pixels, and it happens that the pixels and feature are aligned. The result is a good image of this element. The adjacent set of vertical bars of element 6.3 have the same width and spacing, but apparently are not in good register with the CCD pixels. This demonstrates that resolution is limited by pixel size.

features on the object match the pixels. When the object feature is confined to one pixel and the spacing between features happens to match the pixel size, the apparent image quality is much better, as indicated by an arrow pointing to element 6.3 in Fig. 4(b). The bars and spaces of element 6.3 are each 6.2 microns wide, which is close to the 6.4-micron pixel size. It happens that for the horizontal bars, the features match the pixels and this element is reasonably well resolved, while the larger 6.2 and 6.1 elements, which have different spacings, are not. The 6.3 element consisting of vertical bars is not as well matched feature to pixel as its horizontal companion and is consequently not as clean in appearance.

\section{Demonstration of Four-Color Fluorescence}

Figure 5 shows how cleanly the fluorescence signals from four different dyes with absorption peaks nearly matching the laser wavelengths are separated. These are four images of the same microscopic slide taken with the four lasers turned on in sequence with the imager in configuration a. In taking this image, an initially puzzling artifact appeared. A weaker inverted image ("ghost") of each letter appeared diagonally across from the letter. This was caused by reflection of the fluorescence from the first notch filter traversing back through the first lens and being reimaged on the reflective object plane. The ghost disappears when the notch filters are tilted slightly. This phenomenon is not possible with the imager in configuration $\mathrm{b}$.

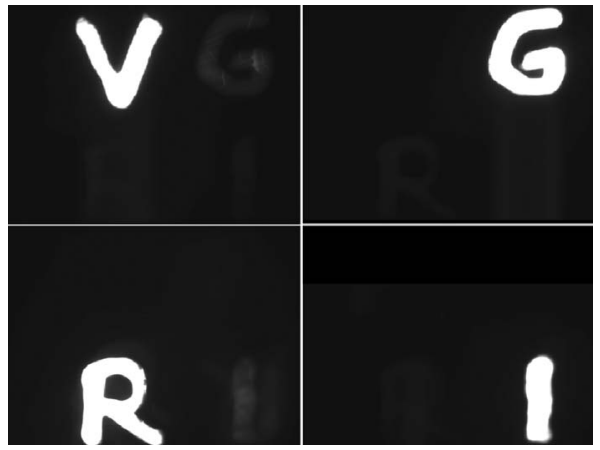

Fig. 5 Fluorescent images of a microscope slide labeled with four different dyes. Each panel covers the full size of the CCD. Coumarin 515 is excited primarily by the 408-nm laser (symbol V); Rh6G is excited primarily by the 532-nm laser (symbol G); LD 700 is excited primarily by the 658-nm laser (symbol R); and IR 125 is excited primarily by the 784-nm laser (symbol I). The dye concentrations were $1 \times 10^{-3} \mathrm{M}$. The lasers are turned on in sequence without any other adjustments. The exposure times are 408 nm, 20 ms; 532 nm, 20 ms; $658 \mathrm{~nm}, 40 \mathrm{~ms}$; and $785 \mathrm{~nm}, 80 \mathrm{~ms}$ (configuration a).

The letters in Fig. 5 are overexposed to the saturation limit of the CCD in order to show the extent to which a laser will cause unwanted excitation of a different dye. Thus, when 408-nm laser exposure completely saturates the violet dye fluorescence, faint images of the fluorescence from the green, red, and deep-red dyes can be seen. When the 532-nm laser exposure completely saturates the green dye fluorescence, a faint image of the red dye fluorescence can be seen. Excitation with the 658-nm laser to red dye fluorescence saturation produces fluorescence from the deep-red dye. Excitation with the 784-nm laser to deep-red dye saturation seems to produce a very weak red dye fluorescence. Excitation by a laser far to the red of the dye peak ordinarily does not produce any of this cross talk because the short-wavelength edge of the dye absorption peak is typically rather sharp.

\section{Example of a Biological Cross Section}

Figure 6 shows two images of the ovary of a young mouse with mutations in Pten and beta-catenin, which result in abnormal development of ovarian follicles that become benign granulosa cell tumors. (For some closely related other work from the Richards Laboratory, see Refs. 19 and 20.) The staining is with hematoxylin and eosin (HE). Figure 6(a) was taken with low-intensity white light; Fig. 6(b) was taken with 532-nm illumination. The different illuminations cause, as might be expected, the gross features to have strikingly different shading in the two images. Figure 6(a) taken in configuration a exhibits vignetting manifested by darker corners.

Similar images can be obtained with a commercial widefield (and thus low-resolution) microscope. However, the ability of this imager to obtain images in fluorescence with independent excitation by four lasers at widely separated wavelengths can provide a new labeling tool. By using four dyes attached to four different targeting agents, it would be possible to achieve on a single slide independently the preferred location of each dye and hence the location of the target of its targeting agent. This could also be done with the rela- 


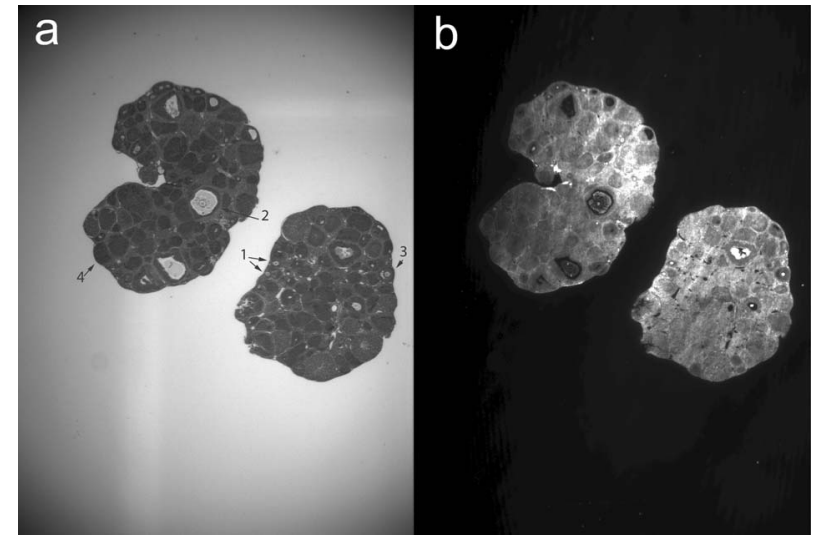

Fig. 6 Images of a section of the ovary of a young mouse with a genetic anomaly, which causes anomalous follicle development. (a) Image taken in white light. (b) Image taken with illumination by the 532-nm laser. Several structures are identified in (a): (1) primary follicle, (2) antral follicle with central feature an oocyte, (3) small follicle with oocyte, and (4) arrow pointing directly at a pretumerous follicle without an oocyte. All the similar dark patches are such pretumerous follicles, and they always lack an oocyte.

tively new four-laser Axon GenePix scanner, which, however, is aimed primarily at high-density microarray scanning.

\section{Microarray Example}

We found that a labeled DNA microarray provides a convenient test of the microscope and its image extraction software by providing a comparison with the image created using a microarray scanner. This comparison illustrates the image processing. In the particular biological experimental procedure used here, RNA was extracted from Arabadopsis thaliana, purified, and characterized. It was then converted to DNA with reverse transcriptase using the Genisphere 3DNA Array 900 W500180 kit. The primer in the reverse transcription process has a characteristic oligonucleotide tail. After the DNA is conjugated to the microarray, a 900 cy 3 dye-labeled dendrimer having an oligo conjugate to the primer tail is allowed to conjugate to the DNA attached to the microarray. The many dye molecules on the dendrimer give a strong fluorescence signal for each original RNA molecule, eliminating the need for polymerase chain reaction (PCR) amplification. Images of the fluorescence from the microarray were taken with $\sim 100 \mathrm{~mW}$ of 532-nm excitation expanded to cover the image area.

Before proceeding further, we performed a local smoothing operation to reduce rapid pixel-to-pixel variations in intensity. A weighted average is computed for each pixel over a small neighborhood of points, and then the pixel value is set equal to that average. In this process, a set of points is fitted to a polynomial in two variables. This is the two-dimensional extension $^{21}$ of the one-dimensional Savitsky-Golay smoothing algorithm.

This image of a portion of the microarray [Figure 7(a)] shows a small number of very brightly fluorescing features; however, many more dimly fluorescing features lie hidden amid the dark background and are indiscernible in this image. Therefore, the range of intensities in the raw images must be truncated in order to bring out the dimmer spots.

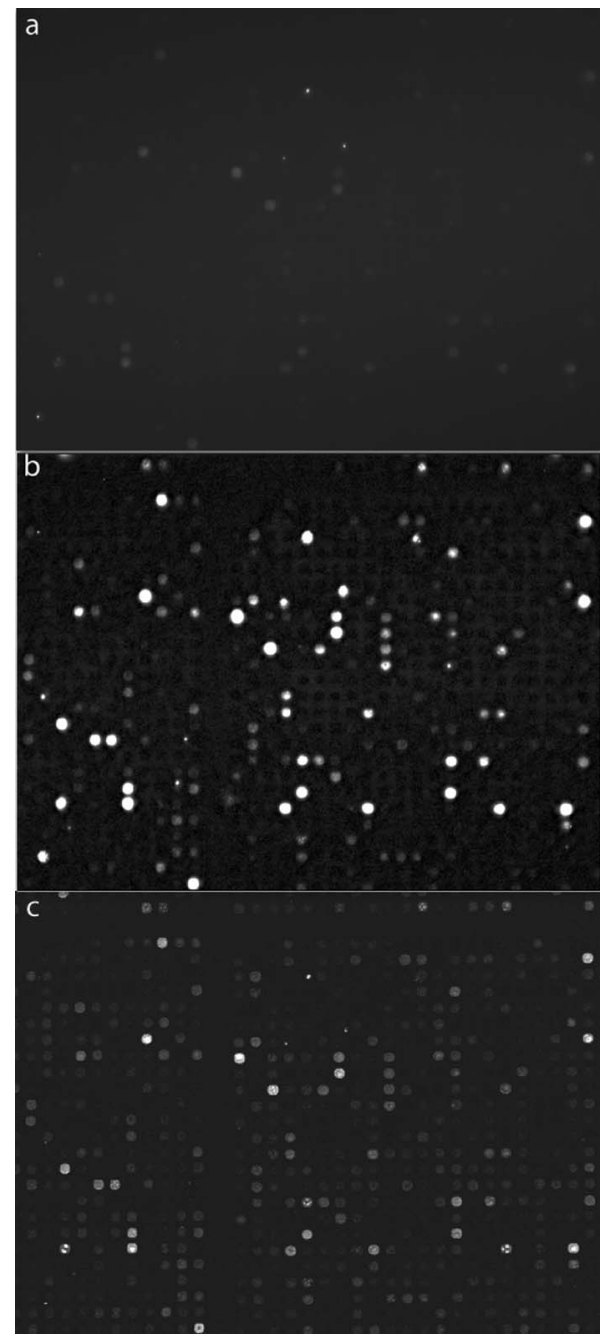

Fig. 7 All three images in this figure cover the full size of the CCD. (a) Raw image obtained with the wide-field fluorescence microscope of a cy 3 labeled DNA array taken with 532-nm excitation. Weaker fluorescence spots cannot be seen because their signals are so small compared with the strongest spots that they fade into the background. The exposure time was $15 \mathrm{~s}$. (configuration b). (b) The same image after smoothing, correction for the variation in background level, and truncation to $3 \%$ of the strongest spot. In addition to the many new spots observed, the basic grid layout of the microarray is revealed because the areas between spots fluoresce weakly. (c) Image of the same area of the microarray illuminated at $532 \mathrm{~nm}$ using a scanning microarray reader. This image has also undergone considerable manipulation to adjust the contrast so that weak spots can be seen.

However, the raw images do not lend themselves directly to truncation, since the object was not uniformly illuminated by the laser. There is a dome-shaped background fluorescence. (This background fluorescence primarily arises because dye adheres to the regions between labeled spots.) The image with peaks must be divided by the background to level the intensity peaks of the features. To obtain a good fitting of the background, we performed a morphological opening ${ }^{22}$ on the images, an operation that removes the peaks while retaining the background.

In order to remove the remaining noise after the opening process, the background treatment was carried one step fur- 


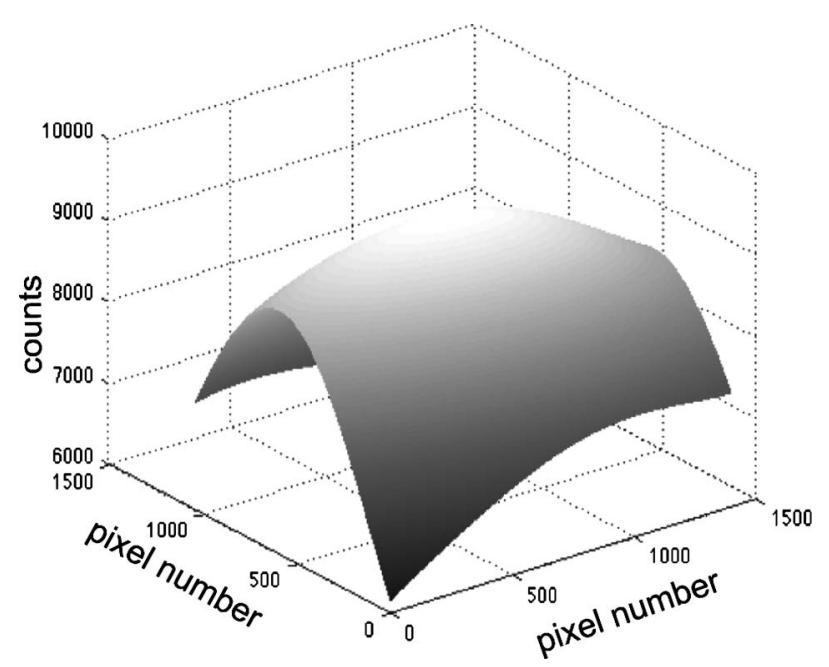

Fig. 8 The result of fitting the background from the opening process with a two-dimensional fifth-order polynomial.

ther by fitting the background from the opening globally with a two-dimensional fifth-order polynomial. Figure 8 shows the resulting background fluorescence for this image as a threedimensional plot.

The 2-D Savitsky-Golay smoothed original was divided by the background and then truncated to bring out features in the block [Fig. 7(b)], even ones apparently not fluorescing. They appear as dark against the fluorescing background grid. The grid pattern that emerges in Fig. 7(b) provides a roadmap for locating the features. This process illustrates how the variation in excitation light intensity intrinsic to oblique illumination can be handled.

Figure $7(\mathrm{c})$ shows the same region of the same microarray created by a scanning fluorescence reader (GenePix 4000B Microarray Scanner). The scanner software also manipulates the image to make fluorescence from dim spots visible in the presence of brightly fluorescing spots. In general, the fluorescent spots in Figs. 7(b) and 7(c) have similar relative intensities. However, careful comparison of Fig. 7(b) with Fig. 7(c) shows that many weak spots that appear in Fig. 7(c) are not seen in Fig. 7(b). Indeed, the fluorescence of the grid in Fig. 7 (b) is stronger than the fluorescence at the missing spots. (Fluorescence from the grid is ignored when the data from the scanner is treated.) The scanner is more sensitive than the wide-field microscope, as there is, in fact, fluorescence from some of the spots that appear dark in Fig. 7(b).

The reader will note that there are various points or small regions in Fig. 7(b) where there is either point fluorescence or fuzzy fluorescent regions, both of which are probably caused by dirt. Most, but not all, of these same points appear in Fig. 7(c). In both images, there are often clues that these bright points are not true biological signals and are usually identifiable as such. Almost none of the fuzzy fluorescent regions appear in Fig. 7(c).

Note that in Fig. 7(a), there are four bright points: three near the center-top of the image forming a triangle and one in the lower-left corner. These are also seen as points in Fig. 7(c), but in Fig. 7(b), they have grown to the size of a microarray feature. However, they are not usually centered properly on the grid. This growth in size is a manifestation of the leakage of electrons from very bright pixels. The apparent change in size of the brightest features in Fig. 7(b) as compared to Fig. 7(a) arises because the severe signal truncation between Fig. 7(a) and Fig. 7(b) used to improve the contrast brings out the electron leakage field around the points.

The scanner has a smaller depth of field than the wide-field microscope. This small depth of field is advantageous in suppressing signal from fluorescence from dirt particles, but occasionally can give rise to falsely high signals for the scanner when the dirt particle happens to be well centered on a feature. A careful look at Fig. 7(c) shows several features containing one or two bright spots, which make ambiguous the calculation of the spot intensity. The same bright spots can be seen in the wide-field image also.

There are several extended regions in Fig. 7(b) that look as though a diaphanous cobweb covers the regions. These do not appear in Fig. 7(c). Whether these fuzzy regions in Fig. 7(b) should be considered an advantage or a defect depends on whether the greater depth of field of this imager happens to be useful or an annoyance in a particular application. The scanner image [Fig. 7(c)] almost has the feel of a mathematical construct, while the wide-field image [Fig. 7(b)] seems more representative of a physical object.

This microarray data provides an opportunity to test whether the imager can provide quantitative data. Careful examination of Figs. 7(b) and 7(c) shows two perpendicular dark alleys running across each of the figures. The vertical alley is centered about $1 / 3$ the distance from the left of the image, and the horizontal alley begins just under a row at the top of the images. These alleys divide the microarray into blocks, with each block containing 26 rows and 25 columns. The registry of the features inside a block is nearly perfect, but there is typically a small offset between blocks, as can be readily noted by comparing two bright features adjacent to each other across an alley. An almost complete block can be seen in the lower-right part of these images. We chose to make quantitative comparisons in this block.

The image consists of a $1040 \times 1392$ matrix of numbers corresponding to the CCD pixels, which need to be matched with the centers of the microarray features in the block. This was done by using a bootstrap procedure to match the features of Fig. 7(b) to the output of the scanner software. This was made easy by the precision of the block grid and the fact that the edges of Fig. 7(b) are very accurately aligned with rows and columns of the microarray features. With the location of the pixel at almost exact center of each feature and the feature size in pixels known, the mean, median, and standard deviation of the fluorescence intensities at each feature could be easily calculated. If there were no possibility of outliers in the distribution of intensities over a feature, the mean would be a more robust measure of the fluorescence intensity. However, if outliers are present, the median should be less affected by them.

Figure 9 shows a plot of the medians of the imager features versus the medians of the scanner features. The intensity of the imager pixels was calculated by dividing the raw pixel value by the background calculated, as described earlier, and then subtracting one to obtain the signal arising from dye fluorescence from the feature. The imager noise levels are about 0.01 to 0.02 . Thus, features with a median imager intensity below 0.03 were not considered. The worst outlier near 


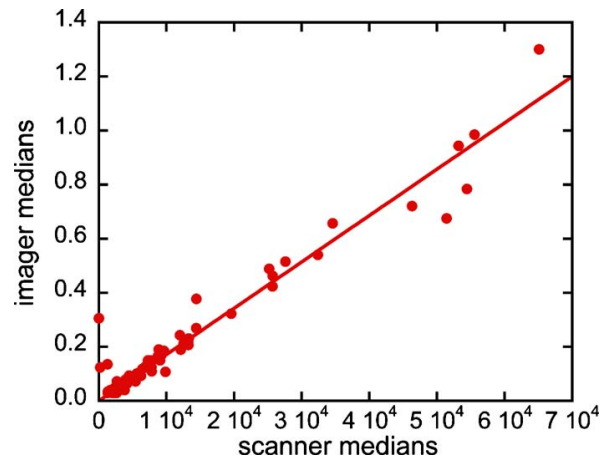

Fig. 9 Plot of median intensity of imager microarray features versus the median intensity of scanner microarray features. The worst looking point at scanner intensity zero and imager intensity 0.3 seems actually to be a glitch in the scanner output with its median value 15 and its mean value 10,200. Even so, the point would be somewhat high.

the 0.3 imager and zero scanner coincides with a large inconsistency in the scanner data, in that it has a much larger mean $(10,000)$ than median $(15)$.

\section{Discussion}

This instrument will not approach the resolution of fluorescence image scanners, and we cannot imagine a modified version of it that could. As explained previously, it has a different role. What makes this instrument different from an ordinary low-power fluorescence microscope is the incorporation of four-laser fluorescence excitation, its greater numerical aperture at this field size, and a relatively low cost. In configuration a, the components of the instrument can be constructed for about $\$ 10,000$ and the instrument assembled in a few weeks. We believe that the professional engineering found in commercial instruments is well worth a greater expenditure. However, if there is no suitable commercial instrument, cost and simplicity of construction become important.

An advantage of this fluorescence microscope in comparison with a scanning system is great flexibility in the objects studied. It will often matter little whether the sample is wet or underwater or dry. Thus, rather complex experiments and objects can be imaged. Most importantly, signals from widely separated parts of the object can be obtained simultaneously, and repeated images can be taken of the whole viewing area within a few seconds. The frequency with which repeated images can be taken is determined by the exposure time per image and the image readout time. Exposure time, which is determined by signal strength and the numerical aperture of the system, can be decreased by using more powerful lasers and faster camera lenses. Image readout time is determined by the speed of the CCD chip and the speed of the AD converter of charge to digits. In the present instrument, it is the $\mathrm{AD}$ converter in the camera that is limiting the rapidity of readout.

There are several ways that the instrument could be improved, but they will involve replacing the $\mathrm{CCD}$ and thus replacing the camera. To improve resolution, a CCD with smaller pixels is needed. To improve field of view while maintaining resolution, a larger CCD is needed. Starlight Express now has a 35-mm cooled camera SXVF-H36 with 7.4 $\times 7.4$ micron pixels at $\$ 7000$. To increase the frame rate, as would be desirable for kinetic studies, a camera with a faster $\mathrm{A} / \mathrm{D}$ converter is needed. The acquisition of repeated images is currently limited by the readout time of the camera $(3.7 \mathrm{~s})$. The CCD itself is capable of being read with full resolution in $1 / 15 \mathrm{~s}$ and at lower resolution in $1 / 60 \mathrm{~s}$.

The geometrical restrictions inherent in oblique illumination prevent uniform illumination. For quantitative work, the illumination profile can be determined, as was described eariler, and the image rescaled by dividing the acquired image by the illumination profile.

\section{Summary}

In summary, the design approach described here offers low cost, great flexibility in treatment of objects, very good sensitivity, and a wide field of view. Data can be obtained across its wide image field with exposures as short as a millisecond. Both improved resolution and a wider field of view would require installing a new (and very probably much more expensive) camera. This imager could find a number of applications, primarily in biological research.

\section{Acknowledgments}

We thank Professor JoAnne Richards for lending us slides of mice ovaries and teaching us how to interpret and understand them. We gratefully acknowledge the help of Professor Bonnie Bartell and her group in providing samples of Arabidopsis thaliana and in teaching us how to extract, purify, and characterize RNA from these samples. We would like to thank Dr. Beth Beason for her advice and the use of the microarray scanning instrumentation. The Arabidopsis microarrays were obtained at a highly subsidized price through the Genome Consortium for Active Teaching, which is directed by Professor A. Malcolm Campbell of Davidson College and supported by the Howard Hughes Foundation. We would also like to thank Professor Robert Sladek of McGill University for providing microarray chips, and we would like to thank Dr. Thomas Albert of NimbleGen Systems for providing some test chips. We would like to thank Gautam Kini from Dr. Biswal's lab for providing the microfluidic channel. This work was supported by The Robert A. Welch Foundation. Wei-Shun Chang was partially supported by a Peter and Ruth Nicholas Fellowship from the Richard E. Smalley Institute of Nanoscale Science and Technology. Heather Barnett, C. Theodore Bucher, and Kevin Daly were supported by undergraduate summer scholarships through a grant from the Camille and Henry Dreyfus Foundation Senior Scientist Mentor Program.

\section{References}

1. E. Haustein and P. Schwille, "Trends in fluorescence imaging and related techniques to unravel biological information," HFSP J. 1, 169-180 (2007).

2. E. L. Botvinick and J. V. Shah, "Laser-based measurements in cell biology," Methods Cell Biol. 82, 81-109 (2007).

3. I. Stayton, Y. Ma, H. Gai, X. Liu, and B. Lin, "Visualizing chemical interactions in life sciences with wide-field fluorescence microscopy toward the single-molecule level," Trends Analyt. Chem. 26(10), 980-992 (2007).

4. V. Levi and E. Gratton, "Exploring dynamics in living cells by tracking single particles," Cell Biochem. Biophys. 48(1), 1-15 (2007).

5. M. Bajenoff and R. N. Germain, "Seeing is believing: a focus on the contribution of microscopic imaging to our understanding of immune system function," Eur. J. Immunol. 37(S1), S18-S33 (2007).

6. S. J. Briddon and S. J. Hill, "Pharmacology under the microscope: the 
use of fluorescence correlation spectroscopy to determine the properties of ligand-receptor complexes," Trends Pharmacol. Sci. 28(12), 637-645 (2007).

7. J. R. Lawrence and T. R. Neu, "Laser scanning microscopy for microbial flocs and particles," IUPAC Ser. Anal. Phys. Chem. Environ. Syst. 10, 469-505 (2007).

8. A. F. Straight, "Fluorescent protein applications in microscopy," Methods Cell Biol. 81, 93-113 (2007).

9. E. M. Sevick-Muraca and J. C. Rasmussen, "Molecular imaging with optics: primer and case for near-infrared fluorescence techniques in personalized medicine," J. Biomed. Opt. 13(4), 041303 (2008).

10. R. Heintzmann and G. Ficz, "Breaking the resolution limit in light microscopy," Methods Cell Biol. 81, 561-580 (2007).

11. J. H. Rice, "Beyond the diffraction limit: far-field fluorescence imaging with ultrahigh resolution," Mol. BioSyst. 3(11), 781-793 (2007).

12. F. L. Labarthet and Y. R. Shen, "Nonlinear optical microscopy," in Optical Imaging and Microscopy, Springer Ser. Opt. Sci. 87, 237-268 (2007).

13. R. A. Cardullo, "Theoretical principles and practical considerations for fluorescence resonance energy transfer microscopy," Methods Cell Biol. 81, 479-494 (2007).

14. M. Oheim, "High-throughput microscopy must reinvent the microscope rather than speed up its functions," Br. J. Pharmacol. 152(1), $1-4$ (2007).

15. R. Sharma, W. Wang, J. C. Rasmussen, A. Joshi, J. P. Houston, K. E.
Adams, A. Cameron, S. Ke, S. Kwon, M. E. Mawad, and E. M. Sevick-Muraca, "Quantitative imaging of lymph function," Am. $J$. Physiol. Heart Circ. Physiol. 292(5), H3109-H3118 (2007).

16. L. D. Lavis and R. T. Raines, "Bright ideas for chemical biology," ACS Chem. Biol. 3(3), 142-155 (2008).

17. A. Mueller-Taubenberger and K. I. Anderson, "Recent advances using green and red fluorescent protein variants," Appl. Microbiol. Biotechnol. 77(1), 1-12 (2007).

18. L. Sampath, W. Wang, and E. M. Sevick-Muraca, "Near-infrared fluorescent optical imaging for nodal staging," J. Biomed. Opt. 13(4), 041312 (2008)

19. D. Boerboom, M. Paquet, M. Hsieh, J. Liu, S. P. Jamin, R. R. Behringer, J. Sirois, M. M. Taketo, and J. S. Richards, "Misregulated Wnt/B-catenin signaling leads to ovarian granulosa cell tumor development," Cancer Res. 65(20), 9206-9215 (2005).

20. M. N. Laguë, M. Paquet, H.-Y. Fan, M. J. Kaartinen, S. Chu, S. P. Jamin, R. R. Behringer, P. J. Fuller, A. Mitchell, M. Doré, L. M. Huneault, J. S. Richards, and D. Boerboom, "Synergistic effects of Pten loss and WNT/CTNNB1 signaling pathway activation in ovarian granulosa cell tumor development and progression," Carcinogenesis 29(11), 2062-2072 (2008).

21. T. R. Edwards, "Two-dimensional convolute integers for analytical instrumentation," Anal. Chem. 54(9), 1519-1524 (1982).

22. K. Reinhard and A. Rosenfeld, Digital Geometry, Morgan Kaufmann, San Franscisco (2004) 